\title{
Erratum to: The utility of clinical features for distinguishing subchondral insufficiency fracture from osteonecrosis of the femoral head
}

\author{
Satoshi Ikemura • Takuaki Yamamoto • \\ Goro Motomura · Yasuharu Nakashima • \\ Taro Mawatari $\cdot$ Yukihide Iwamoto
}

Published online: 29 October 2013

(C) Springer-Verlag Berlin Heidelberg 2013

\section{Erratum to: Arch Orthop Trauma Surg DOI 10.1007/s00402-013-1847-x}

The author would like to correct the error in the publication of the original article. The corrected details are given below for your reading.

The results section of the abstract should read as:

Results The age, proportion of females, the rate of a history of neither corticosteroid intake nor alcohol abuse, and the presence of vertebral compression fracture in subchondral insufficiency fracture were significantly higher than those with osteonecrosis $(p=0.0001,0.0212,0.0001$, and 0.0040 , respectively). ORs for SIF were 12.01 [95\% confidence intervals (CI) 1.35-106.80] and 7.29 (95 \% CI 1.9127.86), if the patient were female and 70 years of age or older, respectively. In addition, OR for SIF was extremely high (OR 56.01, $95 \%$ CI 6.12-512.87) compared to ON, if the patients have a history of neither corticosteroid intake nor alcohol abuse.
The fourth sentence in the second paragraph of the Results section should read as:

There was a significant difference in the rate of a history of neither corticosteroid intake nor alcohol abuse between SIF and $\mathrm{ON}$ patients $(p=0.0001)$.

The Eighth sentence in the second paragraph of the Results section should read as:

Furthermore, OR for SIF was extremely high (OR 56.01, $95 \%$ CI 6.12-512.87) compared to ON, if the patients have a history of neither corticosteroid intake nor alcohol abuse (Table 2).

The first sentence in the Discussion section should read as:

This study first showed the utility of clinical features for the differentiation of SIF from ON, when the patients have a history of neither corticosteroid intake nor alcohol abuse OR for SIF was extremely high (56.01).

The online version of the original article can be found under doi:10.1007/s00402-013-1847-x.

S. Ikemura $\cdot$ T. Yamamoto $(\bowtie) \cdot$ G. Motomura $\cdot$ Y. Nakashima

T. Mawatari $\cdot$ Y. Iwamoto

Department of Orthopaedic Surgery, Graduate School of Medical

Sciences, Kyushu University, 3-1-1 Maidashi, Higashi-ku,

Fukuoka 812-8582, Japan

e-mail: yamataku@ortho.med.kyushu-u.ac.jp 